\title{
Subtipos de adictos a la cocaína con y sin consumo problemático de alcohol asociado: hacia una neuropsicología de la personalidad aplicada a la clínica
}

\section{Subtypes of cocaine addicts with and without associated problematic alcohol use: towards a neuropsychology of personality applied to clinical practice}

\author{
| Eduardo J. Pedrero Pérez*; \\ José M. RUIZ SÁNCHEZ dE LEÓN**
}

\author{
* Centro de Atención a Drogodependientes (CAD 4). Instituto de Adicciones. \\ Madrid Salud. Ayuntamiento de Madrid. \\ ** Dpto. Psicología Básica II (Procesos Cognitivos). Universidad Complutense \\ de Madrid
}

Enviar correspondencia a:

Eduardo J. Pedrero Pérez.

Centro de Atención a Drogodependientes (CAD 4 - San Blas).

C/ Alcalá, 527. 28027 - Madrid.

Teléfono: 9174347 71/72. Fax: 917434775.

E-mail: ejpedrero@yahoo.es

\section{Resumen}

Resulta importante conocer qué factores de personalidad se asocian con la adicción para poder distinguir a los adictos que requieren un tratamiento especializado de quienes no llegan a necesitarlo, asi como diferenciar a los que consiguen la abstinencia de quienes persisten en el consumo a pesar de las consecuencias negativas. El modelo de Cloninger incluye variables biológicas y psicosociales que pueden ser caracterizadas en términos neuropsicológicos. Se analizaron dos muestras, una de sujetos con adicción a cocaína que iniciaban tratamiento $(n=183)$ y otra de población no-clínica ( $n=183)$, equiparadas en sexo, edad y nivel académico. Se controló el abuso/dependencia de alcohol como variable independiente. Se apreciaron diferencias significativas y con gran tamaño del efecto entre los adictos y la población no-clínica en Búsqueda de Novedad y Autodirección, y con menor magnitud, en Evitación del Daño. Estas diferencias se incrementan cuando existe consumo problemático de alcohol añadido. Se establecieron los subgrupos de adictos (clusters) según el perfil de rasgos y se obtuvieron las diferencias en variables como impulsividad funcional/ disfuncional, sintomatología disejecutiva y estrés percibido. Se identificaron 6 subgrupos, algunos de poca gravedad, caracterizándose los más graves por mayores niveles de impulsividad disfuncional, más sintomatología disejecutiva y mayores niveles de estrés percibido. La Autodirección parece reflejar el déficit de los sistemas prefrontales en la regulación de la conducta, asi como en el control emocional y de los impulsos. Se sugiere la evaluación de la personalidad como herramienta más útil que la mera evaluación de síntomas para clasificar a los adictos, determinar sus necesidades y trazar un itinerario terapéutico.

Palabras clave: adicción, cocaina, alcohol, personalidad, impulsividad, corteza prefrontal, estrés percibido, neuropsicología. recibido: Enero 2012 aceptado: Mayo 2012

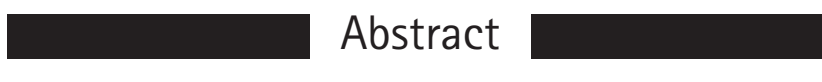

It is important to know which personality factors are associated with addiction so to distinguish addicts that require specialized treatment from those who do not, and to identify those addicts who achieve abstinence from those who continue their substance use despite the negative consequences. Cloninger's model includes biological and psychosocial variables that can be characterized in neuropsychological terms. Two samples were analyzed: individuals who had begun cocaine addiction treatment $(n=183)$ and a non-clinical population sample $(n=183)$, matched for sex, age and educational level. Alcohol abuse/dependence was monitored as an independent variable. Significant differences and large effect size were found between addicts and non-clinical population in Novelty Seeking and Self-Directedness, and to a lesser extent, in Harm Avoidance. These differences increase when problematic use of alcohol is added. According to the profile of traits, clusters of addicts were established and differences were obtained in variables such as functional/ dysfunctional impulsivity, dysexecutive symptoms and perceived stress. Six clusters were identified, some of minor severity, the most severely problematic clusters being characterized by higher levels of dysfunctional impulsivity, more dysexecutive symptoms and higher levels of perceived stress. Self-Directedness seems to reflect the deficit of prefrontal systems in the regulation of behavior, as well as in emotion and impulse control. It is proposed that evaluation of the personality is more useful than the mere assessment of symptoms for classifying addicts, determining their needs and designing a therapeutic itinerary.

Key words: addiction, cocaine, alcohol, personality, impulsiveness, prefrontal cortex, perceived stress, neuropsychology. 
$\mathrm{E}^{2}$ la última década, la cocaína se ha convertido en la droga estimulante consumida con más frecuencia en Europa, siendo la prevalencia en España del 4,4\%, sólo superada por el Reino Unido con el 4,6\% y duplicando la media de todo el continente, 2,1\% (OEDT, 2011). La mayor parte de quienes consumen esta droga tiende a abandonar su uso sin requerir tratamiento específico, incluso habiendo llegado a cumplir criterios para la dependencia (Lopez-Quintero et al., 2011). Sin embargo, un número creciente de personas solicita tratamiento para superar su adicción, más de la mitad de los cuales presenta también consumo problemático de alcohol (OEDT, 2011). El Estudio de Investigación sobre los Resultados del Tratamiento de Drogodependencias (Jones et al., 2009) concluyó que más de la mitad de los pacientes en tratamiento por consumo de cocaína dejaron de consumirla entre tres y cinco meses después de iniciar el tratamiento, persistiendo la abstinencia en el $60 \%$ tras un año completo de tratamiento.

Es de crucial importancia conocer qué rasgos de personalidad se relacionan con la adicción, ya sea como antecedentes, coexistentes o consecuentes, puesto que ello puede informarnos de vulnerabilidades, factores de mantenimiento 0 alteraciones provocadas por el propio proceso adictivo. Cloninger (1986) propuso su modelo biosocial de la personalidad, sustentado en la distinción entre rasgos temperamentales (basados en mecanismos biológicos) y caracteriales (producto del aprendizaje psicosocial), componentes ambos de la personalidad, tanto normal como patológica. Define el temperamento como respuestas automáticas que son en buena parte heredables, se manifiestan desde los momentos iniciales de la vida y son estables en el tiempo. Los rasgos caracteriales representan diferencias individuales en autoconcepto, metas individuales y valores asumidos, que se desarrollan durante la vida a través de la experiencia compartida con el resto de personas. En su última formulación (Cloninger, Svrakic y Przybeck, 1993), se propusieron siete componentes: búsqueda de novedad [BN], evitación del daño [ED], dependencia de recompensa [DR] y persistencia $[P E]$, como rasgos temperamentales; y autodirección [AD], cooperación [CO] y autotrascendencia [AT], como rasgos caracteriales. Este modelo de la personalidad está racionalmente basado en conclusiones sólidas sobre las influencias neurobiológicas y de la experiencia en la estructura de la personalidad y el desarrollo (Cloninger, 2003; Cloninger, Svrakic y Svrakic, 1997). A pesar de recibir algunas críticas por la inconsistencia de su formulación neurobiológica (Paris, 2005), el modelo de Cloninger ha resultado ser de los más útiles en la clínica (Cloninger, 2006), y su instrumento, el $\mathrm{TCl}-\mathrm{R}$, el mejor predictor de trastornos de personalidad (Grucza y Goldberg, 2007).

Estudios precedentes han encontrado que los rasgos del modelo de Cloninger no mostraron relación con la probabilidad de dar positivo en orina en la línea base, en sujetos que iniciaban desintoxicación por cocaína en un hospital (Pérez de los Cobos et al., 2010). En una muestra de adictos a diversas sustancias, emparejados por edad, sexo y nivel de estudios, con sujetos de población no-clínica, aquellos puntuaron significativamente más alto en $\mathrm{BN}$ y más bajo en
AD (Pedrero-Pérez y Rojo-Mota, 2008). La BN ha mostrado relación con deterioro en funcionamiento ejecutivo y toma de decisiones en alcohólicos (Noël et al., 2011) y aparece sistemáticamente elevada en diferentes muestras de adictos, independientemente de la sustancia consumida (Chakroun, Johnson y Swendsen, 2010), mientras que la ED aparece unas veces elevada (Pedrero-Pérez y Rojo-Mota, 2008) y otras baja (Chakroun et al., 2010). La elevada BN asociada a baja DR -personalidades antisociales- parece más vinculadas al consumo de opiáceos, mientras que si se asocia a alta ED -personalidades sensibles- parece más vinculada al abuso de alcohol (Svrakić, Cloninger, Svrakić, Lazić Milivojević y Nastasić, 2010).

La escala de $A D$ ha mostrado relación predictiva sobre toma de decisiones en adictos a opiáceos (Lemenager et al., 2011) y valores bajos se han vinculado estrechamente a la sintomatología frontal en la vida diaria en adictos a diversas sustancias (Ruiz-Sánchez de León et al., 2010), presentando también valores bajos en adicciones sin sustancia (Montag, Jurkiewicz y Reuter, 2010). La AD se ha propuesto como una metavariable de funcionamiento prefrontal, tanto por su formulación conceptual como por sus relaciones empíricas con pruebas de rendimiento neurocognitivo (Pedrero-Pérez et al., 2011b), en la línea de lo propuesto por su propio autor (Cloninger, 2008). Además, una baja AD no sólo aparece vinculada a la adicción, sino a múltiples formas de psicopatología, como los trastornos de la personalidad (Cloninger y Svrakic, 2008), la depresión (Richter y Eisemann, 2002) o la ansiedad (Cloninger, Zohar, Hirschmann y Dahan, 2012), hasta el punto de que un incremento en las puntuaciones de $A D$ se corresponde linealmente con la reducción de los sintomas (Goekoop, De Winter y Goekoop, 2011).

El presente trabajo tiene como objetivo principal estudiar las diferencias en rasgos temperamentales y caracteriales entre sujetos que inician tratamiento por adicción a la cocaína, con y sin consumo problemático de alcohol asociado, y sujetos de población no-clínica. Como objetivo secundario se pretende establecer subgrupos según el perfil de personalidad y explorar las diferencias en cuanto a variables psicológicas relacionadas, como impulsividad, estrés percibido y sintomatología frontal en la vida diaria.

\section{Método}

\section{Participantes}

La muestra estuvo compuesta por 366 sujetos: 183 adictos en tratamiento por abuso/dependencia de cocaína y 183 sujetos de población no-clínica, que cumplimentaron los cuestionarios del estudio. Ambos grupos quedaron emparejados por sexo, edad y nivel académico. La edad media de ambos grupos fue de 32,4 años (d.t.=8,3), en un rango entre 18-53 años. En cada grupo, 111 sujetos eran varones y 72 mujeres. El 3,8\% sólo completaron estudios primarios, el 27,9 \% enseñanza secundaria obligatoria, el $38,3 \%$ secundaria posobligatoria y el $30,1 \%$ contaban con estudios universitarios. 


\section{Instrumentos}

El Inventario del Temperamento y el Carácter-Revisado (TCl-R; Cloninger, 1999), autoinforme de 235 ítems, más 5 de validez, que se responden en una escala Likert de 5 opciones. Mide cuatro dimensiones temperamentales $B N, E D, D R$ y PE- y tres caracteriales $-A D, C O$ y AT-. En el presente trabajo se ha utilizado la versión reducida $\mathrm{TCl}-$ R-67 (Pedrero, 2009a), que consta de las mismas escalas ( 8 ítems cada una) y los 5 ítems de validez; además, se disgregaron de la BN los ítems de Excitabilidad Exploratoria (EE; 6 items), una subescala en el original, al observar que presenta correlaciones sistemáticamente diferentes a los de la escala a la que supuestamente pertenecería. Esta versión ha mostrado adecuadas propiedades psicométricas y una correlación $r>0,80$ con las escalas de la versión original.

La versión española del Cuestionario Disejecutivo (DEXSp; Llanero-Luque et al., 2008), autoinforme elaborado como anexo a la BADS (Burgess, Alderman, Evans, Emslie y Wilson, 1998; Wilson, Alderman, Burgess, Emslie y Evans, 1996), valora sintomatología relacionada con el funcionamiento de la corteza prefrontal en la vida diaria. Consta de 20 ítems que se responden en una escala tipo Likert con 5 opciones entre "nunca" y "con mucha frecuencia". La versión española presentó adecuadas propiedades psicométricas, tanto aplicada a muestras de adictos como a población no-clínica (Pedrero-Pérez et al., 2009, 2011c).

La Escala de Estrés Percibido (EEP; Cohen, Kamarck y Mermelstein, 1983), cuestionario de 14 preguntas sobre los niveles de estrés experimentados en el último mes. La escala, de tipo Likert con 5 opciones entre "nunca" y "siempre", puntúa de 0-56, donde las puntaciones superiores indican mayor estrés percibido. Ha sido validada en población adicta española con adecuados indicadores psicométricos, estructura unifactorial y consistencia interna de $a=0,91$ (Pedrero-Pérez y Olivar-Arroyo, 2010).

El Inventario de Impulsividad de Dickman (1990) consta de 23 ítems divididos en dos subescalas: 11 valoran impulsividad funcional y 12 impulsividad disfuncional. Las respuestas son de elección forzosa en sentido afirmativo o negativo. Aunque existe una versión española validada (Chico, Tous, Lorenzo y Vigil, 2003), para el presente trabajo se ha utilizado una versión modificada, más fiel al original, que ha sido recientemente validada en población adicta (Pedrero-Pérez, 2009b), que mostró una estructura factorial que confirma la existencia de dos subescalas independientes pobremente correlacionadas entre sí $(r=0,10)$ con adecuados valores de consistencia interna ( $a=0,86$ y 0,74 para las subescalas).

Para el diagnóstico de abuso/dependencia se realizaron entrevistas diagnósticas, utilizando criterios DSM-IV-TR. Las pruebas de detección cualitativa de drogas en orina se realizaron mediante inmunoensayos cromatográficos efectuados in situ. La detección de alcohol en aire espirado se realizó mediante alcoholímetro con tecnología analítica dual de célula electroquímica-espectroscopia infrarroja.

\section{Procedimiento}

Los participantes fueron extraídos de la base de datos del CAD-4-San Blas (Instituto de Adicciones. Madrid Salud). La muestra de adictos forma parte de otra mayor, obtenida mediante muestreo consecutivo, de sujetos que inician tratamiento por abuso/dependencia de sustancias. La muestra de población general forma parte de otra mayor, utilizada en estudios de validación, cuya etapa 0 la constituyeron estudiantes de posgrado en Neuropsicología de dos universidades madrileñas; los estudiantes fueron instruidos para recabar la participación de personas de su entorno que cumplieran los siguientes criterios: no haber recibido atención por problemas psiquiátricos o neurológicos, procurar variabilidad en sexo, edad y nivel académico, y estar dispuestos a colaborar de forma anónima y desinteresada. Los estudiantes fueron adiestrados para administrar los cuestionarios y excluir aquellos cuya cumplimentación sugiriera dudas en cuanto a la veracidad, motivación o cumplimiento de instrucciones; finalmente, debieron entregarlas a sus profesores, que supervisaron el cumplimiento de normas. Se excluyeron también los cuestionarios que alcanzaron una puntuación de 20 más respuestas inadecuadas en la escala de validez del cuestionario. Como criterio de inclusión para el presente estudio se buscaron parejas de una y otra muestra que presentaran el mismo sexo, edad y nivel académico. Cuando más de un sujeto coincidía en las variables criterio, se eligió a uno de ellos aleatoriamente. Se obtuvieron así 183 parejas equiparadas.

Los sujetos de la muestra clínica rellenaron los cuestionarios en el curso de una sesión clínica. Para su inclusión se consideró necesario que cumplieran criterios DSM-IV-TR de abuso/dependencia de cocaína, como droga principal en el momento de la evaluación y que llevaran entre 1 y 3 meses abstinentes para toda sustancia no prescrita (constatado mediante controles toxicológicos). Se obtuvieron de su historial clínico edad de inició del consumo, tiempo de adicción, tiempo de abstinencia y gravedad de la adicción (aplicando criterios ASI para alcanzar una única puntuación promedio de todas las dimensiones). Se estableció también un diagnóstico DSM-IV-TR referido al consumo de alcohol, clasificando a los sujetos en no-bebedores, bebedores moderados, abusadores y dependientes (se agrupó a estos dos últimos en la categoría de "bebedores problemáticos"). La abstinencia al alcohol se estableció mediante análisis periódicos no programados de alcohol en aire espirado. La abstinencia a drogas se constató mediante análisis toxicológicos programados (una vez a la semana) y no programados. Todos los participantes fueron informados del doble objetivo de la evaluación (clínico y de investigación) y firmaron consentimiento para el uso anónimo de los resultados.

\section{Análisis de datos}

Se efectuó una prueba de Levene que reveló heterogeneidad en las varianzas de las puntuaciones entre ambas muestras en 5 de las 8 dimensiones. Por ello, se optó por un análisis de varianza a partir de la prueba robusta de Brown-Forsythe. Para calcular el tamaño del efecto de las 
Tabla 1. Diferencias entre las puntuaciones medias obtenidas por adictos $(n=183)$ y población no-clínica $(n=183)$ en el TCl-R-67.

\begin{tabular}{cccccccccc}
\hline & \multicolumn{3}{c}{ No-clínica } & \multicolumn{3}{c}{ Adictos } & & & \\
& Media & D.T. & Media & D.T. & B-F & Sig. & $d$ & $r_{d}$ \\
\hline Búsqueda de Novedad & 18,16 & 5,01 & 26,78 & 6,88 & 187,93 & $<0,001$ & 1,43 & 0,58 \\
Evitación del Daño & 19,98 & 6,13 & 24,10 & 6,55 & 38,64 & $<0,001$ & 0,65 & 0,31 \\
Dependencia de Recompensa & 27,46 & 7,11 & 26,93 & 8,86 & 0,39 & 0,53 & 0,07 & 0,03 \\
Persistencia & 27,92 & 5,95 & 25,84 & 7,03 & 9,36 & $<0,01$ & 0,32 & 0,16 \\
Excitabilidad Exploratoria & 20,95 & 4,35 & 21,26 & 4,44 & 0,42 & 0,52 & 0,07 & 0,04 \\
Autodirección & 31,90 & 5,98 & 24,28 & 7,13 & 122,63 & $<0,001$ & 1,16 & 0,50 \\
Cooperación & 31,21 & 5,53 & 29,99 & 6,29 & 3,87 & 0,50 & 0,21 & 0,10 \\
Autotrascendencia & 17,27 & 6,58 & 18,39 & 6,74 & 10,41 & $<0,01$ & 0,17 & 0,08 \\
\hline
\end{tabular}

Nota: B-F=Estadistico de Brown-Forsythe para dos muestras independientes con varianzas no homogéneas; $d$ =estadístico de Cohen; $r_{d}=$ transformación de la d de Cohen en coeficiente de correlación.

Tabla 2. Diferencias entre las puntuaciones medias obtenidas por adictos a cocaína con $(n=95)$ y sin problemas de alcohol asociados (abuso o dependencia; $\mathbf{n = 8 8}$ ) en el TCl-R-67.

\begin{tabular}{ccccccccc}
\hline & \multicolumn{7}{c}{ Cocaina } & \multicolumn{7}{c}{ Cocaina + Alcohol } & & & & \\
& Media & D.T. & Media & D.T. & B-F & Sig. & d & $r_{d}$ \\
\hline Búsqueda de Novedad & 24,68 & 6,97 & 28,73 & 6,22 & 17,06 & $<0,001$ & 0,61 & 0,29 \\
Evitación del Daño & 22,26 & 7,07 & 25,80 & 5,53 & 14,06 & $<0,001$ & 0,56 & 0,27 \\
Dependencia de Recompensa & 26,40 & 9,26 & 27,43 & 8,48 & 0,62 & 0,43 & 0,12 & 0,06 \\
Persistencia & 26,06 & 7,30 & 25,63 & 6,80 & 0,17 & 0,68 & 0,06 & 0,03 \\
Excitabilidad Exploratoria & 21,33 & 4,62 & 21,19 & 4,29 & 0,05 & 0,83 & 0,03 & 0,02 \\
Autodirección & 26,32 & 7,25 & 22,40 & 6,51 & 14,71 & $<0,001$ & 0,57 & 0,27 \\
Cooperación & 30,81 & 6,28 & 29,23 & 6,25 & 2,89 & 0,09 & 0,25 & 0,13 \\
Autotrascendencia & 18,16 & 6,50 & 20,77 & 6,74 & 7,10 & $<0,01$ & 0,39 & 0,19 \\
\hline
\end{tabular}

Nota: $\mathrm{B}-\mathrm{F}=E$ Estadistico de Brown-Forsythe para dos muestras independientes con varianzas no homogéneas; $\mathrm{d}=$ =stadistico de Cohen para estimación del tamaño del efecto; $r_{\mathrm{d}}=$ transformación de la d de Cohen en coeficiente de correlación.

diferencias se utilizó el estadístico $d$ de Cohen y su transformación en coeficiente de correlación. Se realizaron pruebas de correlación lineal utilizando la $r$ de Pearson y se utilizó la corrección de Bonferroni para comparaciones múltiples, para evitar la comisión del error tipo I. Se efectuó un análisis de conglomerados sobre las puntuaciones del TCl-R-67 en dos pasos: primero, se estimó el número idóneo de agrupaciones mediante análisis jerárquico, utilizando el método de Ward (que genera conglomerados con varianza mínima dentro de ellos y máxima entre ellos) y la distancia euclídea al cuadrado (para conseguir la mínima perdida de información y la máxima similaridad de los elementos de cada conglomerado); posteriormente, se forzó una solución con el número ideal de agrupaciones encontrado, clasificando a todos los sujetos en alguna de ellas y estimando las puntuaciones medias de las variables de agrupación y las variables criterio. Las puntuaciones de las pruebas se normalizaron y se transformaron en una distribución de media 50 y d.t. 10, a partir de las puntuaciones obtenidas en la muestra de población no-clínica, para facilitar su interpretación gráfica.

\section{Resultados}

Aparecieron diferencias significativas, con gran tamaño del efecto, en las dimensiones de $B N$ y $A D$, entre adictos $y$ controles, con moderado tamaño en ED, y con bajo tamaño en PE y AT (Tabla 1).

Cuando se compararon los resultados obtenidos por los adictos a cocaína, con o sin consumo problemático de alcohol asociado, aparecieron diferencias significativas en las mismas variables (salvo PE) y en el mismo sentido que en el 
Tabla 3. Correlaciones entre rasgos y variables relativas a la adicción.

\begin{tabular}{llllll}
\hline & $\begin{array}{l}\text { C.l. } \\
\text { premórbido }\end{array}$ & $\begin{array}{l}\text { Tiempo de } \\
\text { Abstinencia }\end{array}$ & Gravedad & $\begin{array}{l}\text { Tiempo de } \\
\text { Adicción }\end{array}$ & $\begin{array}{l}\text { Edad de } \\
\text { inicio }\end{array}$ \\
\hline Búsqueda de Novedad & $-0,08$ & $-0,15^{*}$ & $0,23^{* * *}$ & 0,06 & $-0,13^{*}$ \\
Evitación del Daño & $-0,07$ & 0,09 & $0,22^{* * *}$ & $-0,13^{*}$ & 0,09 \\
Dependencia de Recompensa & $-0,08$ & $-0,17^{*}$ & $0,31^{* * *}$ & $0,16^{*}$ & $-0,16^{*}$ \\
Persistencia & $-0,03$ & 0,11 & $-0,20^{*}$ & $-0,15^{*}$ & $0,14^{*}$ \\
Excitabilidad Exploratoria & $-0,02$ & 0,02 & $-0,09$ & 0,02 & $0,13^{*}$ \\
Autodirección & 0,04 & 0,08 & $-0,23^{* * *}$ & 0,08 & $-0,04$ \\
Cooperación & 0,07 & $-0,11$ & $-0,01$ & 0,10 & 0,00 \\
Autotrascendencia & $-0,01$ & $-0,04$ & 0,07 & $0,27^{* * *}$ & $-0,10$ \\
\hline
\end{tabular}

Nota: ${ }^{* * *} p<0,001 ; * * 0,01 ; i p<0,05$; en negrita correlaciones significativas tras efectuar la corrección de Bonferroni $(p<0,00125)$.

Tabla 4. Correlaciones entre las escalas del TCI-R-67 y las del DII, EEP y DEX.

\begin{tabular}{lllllllll}
\hline & BN & ED & DR & PE & EE & AD & CO & AT \\
\hline Impulsividad disfuncional & $0,71^{*}$ & $0,38^{*}$ & 0,02 & $-0,23$ & $-0,04$ & $-0,58^{*}$ & $-0,22$ & 0,14 \\
Impulsividad funcional & 0,17 & $-0,51^{*}$ & 0,25 & 0,25 & $0,47^{*}$ & $0,29^{*}$ & 0,02 & $-0,01$ \\
Estrés percibido & 0,31 & $0,43^{*}$ & $-0,05$ & $-0,31$ & 0,10 & $-0,52^{*}$ & $-0,02$ & $-0,15$ \\
Sintomatología disejecutiva & $0,60^{*}$ & $0,65^{*}$ & $-0,19$ & $-0,35^{*}$ & $-0,23$ & $-0,73^{*}$ & $-0,31^{*}$ & $0,30^{*}$ \\
\hline
\end{tabular}

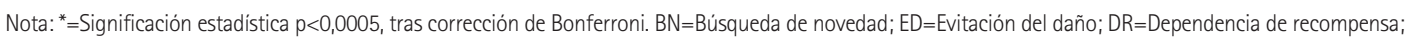
$\mathrm{PE}=$ Persistencia; $\mathrm{AD}=$ Autodirección; $\mathrm{CO}=$ Cooperatividad; $\mathrm{AT}=$ Autotrascendencia.

caso anterior (mayores puntuaciones en AD, ED y AT, y menores en $A D$ en el grupo de policonsumidores; Tabla 2).

Seguidamente se exploró la relación entre rasgos de personalidad y variables relativas a la adicción: gravedad, tiempo de abstinencia alcanzado, tiempo de consumo continuado y la edad de inicio de ese consumo (Tabla 3 ).

Para poder caracterizar más adecuadamente estas diferencias, se exploró la relación entre los rasgos medidos por el TCl-R-67 y otras variables psicológicas. En la Tabla 4 se observa el amplio patrón correlacional, especialmente marcado en las variables que se mostraron críticas en las diferencias entre grupos. La $A D$ muestra relación significativa (una vez eliminado el efecto del azar) y con considerable tamaño del efecto con casi todas las variables psicológicas, mientras que la AT no parece estar relacionada con ninguna, salvo la sintomatología de origen frontal.

Puesto que el patrón de correlaciones entre los rasgos y las variables psicológicas mostraba una gran similitud, se efectuó un diagnóstico de multicolinealidad para conocer la redundancia entre los rasgos. No apareció colinealidad entre las escalas de rasgos (Factor de Inflación de la Varianza $\mathrm{FIV}<1,5$; Tolerancia $>0,70$; Autovalor $=0,12$; Índice de Condición $I C=19,78)$. La varianza de la $A D$ podía predecirse en un $66,3 \%$ a partir de las cuatro dimensiones temperamentales: $E D\left(R^{2}=0,55 ; \beta=-0,55\right), B N\left(R^{2}=0,10 ; \beta=-0,33\right), P E$ $\left(R^{2}=0,01 ; \beta=0,13\right)$ y $D R\left(R^{2}=0,003 ; \beta=0,07\right)$.
Finalmente, se efectuó un análisis de clusters sobre las puntuaciones en rasgos de la muestra completa. Se realizó, en primer lugar, un análisis jerárquico para obtener el número idóneo de conglomerados; se obtuvo una solución de 6 clusters. Posteriormente, se forzó esta solución y se estudiaron las puntuaciones obtenidas por los sujetos asignados a cada uno de ellos en otras pruebas. Se obtuvieron las puntuaciones medias en variables relacionadas con la adicción, así como los porcentajes de cada muestra adjudicados a cada cluster y se estableció el porcentaje de sujetos adictos que mostraba consumo problemático de alcohol añadido; los resultados se muestran en la Tabla 5.

\section{Discusión}

Los resultados encuentran diferencias significativas y con considerable tamaño del efecto entre los consumidores de cocaína que inician tratamiento y los sujetos de población general en dos dimensiones de personalidad: mayores puntuaciones en las escalas de BN y ED, y menores en la de AD. Estas diferencias son más acusadas cuando se añade consumo problemático de alcohol. Estos resultados son, en su mayor parte, coherentes con lo encontrado en la literatura revisada (ej., Chakroun et al., 2010).

En términos neuropsicológicos la interpretación de estos datos apunta a una pérdida de control prefrontal (baja AD) 
Tabla 5. Puntuaciones de cada cluster en las dimensiones del TCl- $\mathrm{R}$, porcentaje de sujetos de cada submuestra incluidos en cada cluster, variables de consumo, sintomatología frontal e impulsividad.

\begin{tabular}{|c|c|c|c|c|c|c|}
\hline & \multicolumn{6}{|c|}{ Clusters } \\
\hline & 1 & 2 & 3 & 4 & 5 & 6 \\
\hline Búsqueda de Novedad & $=$ & $=$ & $=$ & $\uparrow$ & $\uparrow \uparrow$ & $\uparrow \uparrow$ \\
\hline Evitación del Daño & $=$ & $=$ & $=$ & $=$ & $\uparrow$ & $\uparrow$ \\
\hline Dependencia de Recompensa & $=$ & $=$ & $\downarrow \downarrow$ & $=$ & $=$ & $\downarrow$ \\
\hline Persistencia & $=$ & $=$ & $=$ & $=$ & $\downarrow$ & $\downarrow$ \\
\hline Excitabilidad Exploratoria & $=$ & $=$ & $=$ & $=$ & $=$ & $=$ \\
\hline Autodirección & $=$ & $=$ & $=$ & $=$ & $\downarrow \downarrow$ & $\downarrow \downarrow$ \\
\hline Cooperación & $=$ & $=$ & $=$ & $=$ & $=$ & $=$ \\
\hline Autotrascendencia & $=$ & $\uparrow$ & $=$ & $=$ & $=$ & $=$ \\
\hline$\%$ No-clinica & 37,9 & 17,2 & 29,6 & 11,2 & 3,0 & 1,2 \\
\hline \%Adictos & 8,2 & 12,0 & 21,3 & 20,2 & 21,9 & 16,4 \\
\hline \%Consumo problemático alcohol & 0 & 27,8 & 64,9 & 61,3 & 80,0 & 95,0 \\
\hline Gravedad & 5,50 & 6,13 & 5,42 & 6,15 & 7,00 & 6,86 \\
\hline Tiempo de adicción & 4,75 & 13,63 & 9,08 & 7,17 & 11,29 & 6,86 \\
\hline Edad de inicio & 24,75 & 21,38 & 23,75 & 20,67 & 21,00 & 20,43 \\
\hline Sintomatología frontal & $=$ & $=$ & $=$ & $=$ & $\uparrow \uparrow$ & $\uparrow \uparrow$ \\
\hline Impulsividad disfuncional & $=$ & $=$ & $=$ & $\uparrow$ & $\uparrow \uparrow$ & $\uparrow \uparrow$ \\
\hline Impulsividad funcional & $=$ & $=$ & $=$ & $=$ & $=$ & $=$ \\
\hline Estrés percibido & $=$ & $=$ & $\uparrow$ & $=$ & $\uparrow$ & $\uparrow$ \\
\hline
\end{tabular}

Nota: $=$ puntuaciones en la media \pm 1 d.t. $\uparrow$ y $\downarrow$ = puntuaciones entre 1 d.t. $<x<2$ d.t, por encima y por debajo de la media poblacional; $\uparrow \uparrow$ y $\downarrow \downarrow=$ puntuaciones entre $x>2$ d.t, por encima y por debajo de la media poblacional.

que posibilita la alternancia de conductas de aproximación de carácter impulsivo, no orientadas a metas, posiblemente basadas en la actividad del circuito corticoestriatal no regulado (alta BN) y de conductas guiadas por inputs emocionales, procedentes de la amígdala, que tampoco pueden ser reguladas por el cortex prefrontal (alta ED). Estos resultados son congruentes con el modelo triádico de la conducta motivada (Ernst y Fudge, 2009) y con los hallazgos habituales en sujetos con adicción a diversas sustancias o sin sustancias, de modo que más que por una predisposición biológica a la BN o a la conducta impulsiva, la adicción se explicaría por una incapacidad para desarrollar los mecanismos de control propios de la corteza prefrontal, tanto los de carácter impulsivo como los de carácter emocional, incapacidad que se vería agravada por la propia adicción (Pedrero-Pérez, LópezDurán y Olivar-Arroyo, 2011).

Tanto la BN como la ED y la AD muestran relaciones significativas con la gravedad del proceso adictivo. Pero también aparece relación con elevados niveles de DR. La DR sería la tendencia a responder intensamente a señales de grati- ficación y a mantener o resistir la extinción de conductas que previamente fueron asociadas con gratificación o evitación del castigo. Esto supone que el sujeto con altos niveles de DR tiene importantes dificultades para aprender nuevas conductas porque el proceso de aprendizaje asociativo está interrumpido, lo que mantiene conductas que fueron reforzadas previamente, aunque ya no lo sean. No obstante, esta característica no diferencia a sujetos adictos de los que no lo son, ni se ve afectada por el consumo problemático de alcohol concurrente; parece, pues, que la alta DR no favorecería la adicción, pero sí posiblemente su persistencia, a pesar de las consecuencias negativas. Este hecho debe ser tenido en cuenta en los procesos de rehabilitación, considerando que los sujetos con altos niveles en DR van a requerir mayores dosis de aprobación y reforzamiento ante los logros que se vayan produciendo.

La única dimensión afectada por el tiempo de adicción es la AT, que agrupa características de espiritualidad, misticismo, pensamiento mágico y religioso, y se relaciona con la imaginación y la capacidad del sujeto para aceptar la 
ambigüedad y la incertidumbre. Su influencia en la conducta parece depender de la combinación del resto de dimensiones caracteriales (Svrakic et al., 2002). Cabe pensar que a mayor tiempo de adicción se incrementa la probabilidad de interpretar la realidad y resolver la incertidumbre a partir de explicaciones de tipo mágico, místico o irracional. Esta característica sí diferenció a los adictos de la población general, incrementándose la diferencia cuando existía abuso concurrente de alcohol, pero el tamaño del efecto resultó ser muy bajo.

No se observan relaciones significativas con el tiempo de abstinencia, aunque no era esperable su aparición dado que el periodo entre 1 y 3 meses de abstinencia es insuficiente para consolidar cambios significativos. Tampoco aparecen relaciones significativas entre edad de inicio en el consumo problemático de cocaína y rasgos de personalidad: en este caso, la interpretación sugiere que el punto en el que se estableció la adicción es arbitrario en un continuo en el que el consumo previo de ésta y otras sustancias marca un trazado complejo.

La correlación entre impulsividad disfuncional y BN sugiere que ambas están midiendo esencialmente lo mismo. En términos neuropsicológicos, la impulsividad disfuncional aparece como una disposición que dificulta la realización de las tareas de forma global, sin interferencia específica sobre alguna función; se relaciona con una tendencia a la actuación irreflexiva que probablemente interfiera con la comprensión de las instrucciones, la planificación de la acción y la dificultad para mantener la atención durante su desarrollo (Pedrero-Pérez, Ruiz-Sánchez de León, Rojo-Mota, LlaneroLuque y Puerta-García, 2012). Esa interferencia global parecería corresponder con lo que Goldman (1990) denominó "neblina cognitiva" ("cognitive haze"), estado ligeramente confusional con deterioro en las capacidades de atención, comprensión y memoria, que presentan los adictos en las semanas posteriores a la consecución de la abstinencia. La correlación de la impulsividad disfuncional no sólo con la BN, sino también con altos niveles de ED y bajos de $A D$ vuelve a sugerir la explicación triádica (Ernst y Fudge, 2009), de modo que podrian ser los fallos en la regulación córtico-prefrontal los responsables de la "neblina cognitiva", la conducta impulsiva y la desregulación emocional. Por el contrario, la impulsividad funcional presenta correlaciones opuestas, apuntando a una conducta orientada a metas, caracterizada por un procesamiento rápido, basado en una adecuada gestión atencional (Pedrero-Pérez et al., 2012).

Todas estas relaciones se concretan al observar el patrón correlacional entre la sintomatología disejecutiva y los rasgos del modelo de Cloninger. En efecto, sintomatología disejecutiva parece ser sinónimo de baja $A D$, y se corresponde con conducta impulsiva (alta BN), descontrol emocional (alta $E D$ ), inconstancia en tareas con gratificación demorada (baja $P E)$, desinterés por las necesidades ajenas (baja CO) y preferencia por explicaciones mágicas o irracionales para resolver la incertidumbre (alta AT).

Un elemento adicional debe ser tenido en cuenta: el estrés percibido. Su elevada correlación negativa con AD y positiva con ED sugiere que puede ser unos de los elementos claves en el fallo prefrontal subyacente al resto de alteraciones. Contamos con abundante evidencia de que el estrés agudo provoca un deterioro significativo, aunque reversible, del funcionamiento prefrontal, en modelos animales (Liston, McEwen y Casey, 2009) y en estudios con humanos (Arnsten, 2009) y también con propuestas que sugieren su relación con los procesos de adquisición, mantenimiento y abandono/recaída de las conductas adictivas (Schwabe et al., 2011). Estas alteraciones inducidas por el estrés pueden estar también en la base de muchas de las condiciones psicopatológicas diagnosticadas en el inicio del tratamiento de los adictos, como los trastornos del estado de ánimo o de la personalidad (Reich, 2002), que desaparecen rápidamente y son tenidos erróneamente por "patología dual" (Pedrero-Pérez, LópezDurán y Olivar-Arroyo, 2011).

Finalmente, se ha clasificado a los sujetos participantes en función de los rasgos del modelo de Cloninger y se han estudiado los correlatos que caracterizan a cada grupo. Se observa (Tabla 5) que los dos primeros grupos no presentan rasgos característicos, salvo una cierta elevación de la AT en el primero de ellos. En estos dos grupos queda integrado el $55 \%$ de los sujetos de población no-clínica y sólo el $20 \%$ de los adictos, quienes, además, presentan en pocos casos (en ninguno del primer grupo) un consumo problemático de alcohol añadido. El primer grupo está integrado por los sujetos que más tarde se iniciaron en el consumo problemático de cocaína, llevan menos tiempo manteniéndolo y presentan menores indicadores de gravedad. El segundo grupo, en cambio, presenta el mayor tiempo de consumo e indicadores medios de gravedad; posiblemente se trata de los sujetos que han conseguido un consumo más adaptativo, siendo difícil interpretar el verdadero significado de esa elevada AT diferencial. Ambos grupos podrían representar a aquellos sujetos que alcanzan con facilidad la abstinencia, la mantienen y no muestran excesivos efectos residuales de su consumo previo (Jones et al., 2009).

El tercer grupo se caracteriza por una baja DR, propia de personalidades poco sociables, bien representado tanto entre población no-clínica (30\%) como entre adictos (21\%), quienes muestran leves indicadores de gravedad, inicio tardio del consumo problemático y elevada proporción de consumo problemático de alcohol concurrente. Los altos niveles de estrés percibido parecen sugerir que el abuso de cocaína y alcohol representan modalidades de escape o evitación encaminadas a reducir el malestar. El cuarto grupo, en el que se encuentra la quinta parte de los adictos y sólo la décima parte de la muestra no-clínica, se caracteriza, en cambio, por niveles moderadamente altos de BN, así como de impulsividad disfuncional; el grupo de adictos presenta, además, un importante consumo de alcohol y una edad muy baja de inicio en la adicción a la cocaína, presumiblemente precedido por un consumo excesivo de alcohol. Posiblemente representen a un grupo que, a diferencia del anterior, busca gratificación en el consumo sin cálculo de las consecuencias.

Los grupos 5 y 6 representan los niveles más elevados de gravedad. Apenas se encuentra entre ellos un 4\% de la población no-clínica, en tanto que en ellos se agrupa el 38\% de los adictos. Presentan en común la triada ya comenta- 
da: alta $B N$, alta $E D$ y baja $A D$, además de baja $P E$; la única diferencia entre estos dos grupos es el bajo nivel de DR en el último de ellos (sugiriendo un patrón de comportamiento antisocial; Svrakic et al., 2002). A la elevada impulsividad disfuncional se añaden indicadores de severo deterioro de origen frontal en las actividades cotidianas, así como un importante nivel de estrés percibido. Es muy probable que sean éstos los sujetos que presenten criterios para diagnósticos concurrentes al de su adicción (no sólo a la cocaína, sino también al alcohol), que probablemente no representaran en la mayor parte de los casos procesos independientes, sino fallos globales en sus capacidades para el control emocional, la toma de decisiones y la programación de su conducta. Estos fallos, a su vez, generarían malas consecuencias en su entorno, que incrementarian el estrés y el consumo de sustancias, en una espiral bien conocida en los programas de tratamiento. Es destacable que el sexto grupo presenta un tiempo de adicción a la cocaína relativamente breve, aunque el 95\% de ellos presentan adicción al alcohol, probablemente previa, por lo que sería necesario conocer la funcionalidad de cada sustancia en el patrón adictivo general.

A partir de los rasgos temperamentales y caracteriales se ha podido clasificar a los sujetos adictos en diversos grupos. Si los dos primeros representan procesos adictivos leves, los dos siguientes podrían representar grupos de riesgo para el paso a los perfiles claramente patológicos. Los dos perfiles graves presentan como particularidad principal un patrón caracterizado por fallo en los mecanismos de control ejecutivo frontal (tanto metacognitivos como emocionales; Ardila, 2008), que probablemente se traduce en una incapacidad para determinar las propias metas y organizar la propia conducta. Estos perfiles patológicos son los principales candidatos a beneficiarse de tratamientos completos, que incluyan, al menos en sus fases iniciales, estrategias de rehabilitación cognitiva para las funciones afectadas (Pedrero-Pérez, RojoMota, Ruiz-Sánchez de León, Llanero-Luque y Puerta-García, 2011; Rojo-Mota, Iraurgi-Castillo y Sánchez-Cabeza, 2011). Cabe suponer que estos perfiles pueden representar a personas con determinadas predisposiciones genéticas que, en determinado contexto evolutivo, no han sido capaces de aprender a gestionar las demandas ambientales, sus propias capacidades y su relación con las drogas, a las que han atribuido determinadas funciones y expectativas, siendo incapaces de mantener el control, y cuyos déficits se han agravado por el propio proceso adictivo y sus consecuencias (Schwabe et al., 2011). Por ello, la detección precoz de estos perfiles puede favorecer una intervención temprana de carácter preventivo, evitando el deterioro añadido por el propio contacto con las sustancias, favoreciendo el desarrollo adecuado de estrategias de control cognitivo y emocional desde el currículo educativo (Olivar-Arroyo, 2011).

La principal limitación del presente estudio se sitúa en la representatividad de ambas submuestras. Sin embargo, es preciso hacer constar que el objetivo del estudio no se situaba en la representatividad, sino en la relación entre variables. La equiparación ("matching") en variables sociodemográficas es uno de los métodos más adecuados para eliminar interferencias en el establecimiento de la relación entre variables
(Rosenbaum, 1989), si bien es preciso realizar estudios en medios diferentes para confirmar las relaciones encontradas en este trabajo.

En conclusión, los rasgos de temperamento y carácter propuestos por Cloninger pueden ser de utilidad para clasificar a los adictos a cocaína y determinar sus necesidades terapéuticas con más precisión que la mera clasificación sintomatológica. Esta clasificación en función de los perfiles de personalidad puede dar cuenta, además, de las alteraciones cerebrales que están en la base de la adicción, así como de buena parte de los trastornos coexistentes. La evaluación de la personalidad, junto con la evaluación neuropsicológica, son ingredientes de gran interés para el futuro de los programas de tratamiento de sujetos adictos a cocaína, así como a otras sustancias e incluso de las denominadas adicciones $\sin$ sustancia.

\section{Conflicto de intereses}

Los autores declaran no tener ningún conflicto de intereses en esta publicación.

\section{Referencias}

Ardila, A. (2008). On the evolutionary origins of executive functions. Brain and Cognition, 68, 92-99. doi:10.1016/j.bandc.2008.03.003

Arnsten, A. F. T. (2009). Stress signalling pathways that impair prefrontal cortex structure and function. Nature Reviews Neuroscience, 10, 410-422. doi:10.1038/nrn2648

Burgess, P. W., Alderman, N., Evans, J., Emslie, H. y Wilson, B. (1998). The ecological validity of tests of executive function. Journal of International Neuropsychological Society, 4, 547-558.

Chakroun, N., Johnson, E. I. y Swendsen, J. (2010). Mood and personality-based models of substance use. Psychology of Addictive Behaviors, 24, 129-136. doi: 10.1037/a0018184

Chico, E., Tous, J. M., Lorenzo, U. y Vigil, A. (2003). Spanish adaptation of Dickman's impulsivity inventory: Its relationship to Eysenck's personality questionnaire. Personality and Individual Differences, 35, 1883-1892. doi: 10.1016/S0191-8869(03)00037-0

Cloninger, C. R. (1986). A unified biosocial theory of personality and its role in the development of anxiety states. Psychiatric Developments, 3, 167-226.

Cloninger, C. R. (1999). The Temperament and Character InventoryRevised. St Louis, MO: Center for Psychobiology of Personality, Washington University.

Cloninger, C. R. (2003). Completing the psychobiological architecture of human personality development: temperament, character and coherence. En: Ursula M, Lindenberger, U (eds). Understanding human development: dialogues with lifespan psychology (pp.159-181). Dordrecht: Kluwer.

Cloninger, C. R. (2006). Commentary on Paris: Personality as a dynamic psychobiological system. En T.A. Widiger, E. Simonsen, P. Sirovatka y D.A. Regier (Eds.), Dimensional models of personality 
disorders: Refining the Research Agenda for DSM-V (pp. 73-76). Washington DC: American Psychiatric Publishing.

Cloninger, C. R. (2008). The psychobiological theory of temperament and character: Comment on Farmer and Goldberg (2008). Psychological Assessment, 20, 292-299. doi: 10.1037/a0012933

Cloninger, C. R. y Svrakic, D. M. (2008). Personality disorders. En S. H. Fatemi y P. J. Clayton (eds.), The medical basis of psychiatry. Part III (pp. 471-483). Totowa NJ: Humana Press. doi: 10.1007/978-159745-252-6_28

Cloninger, C. R., Svrakic, D. M. y Przybeck, T. R. (1993). A psychobiological model of temperament and character. Archives of General Psychiatry, 50, 975-990.

Cloninger, C. R., Svrakic, N. M. y Svrakic, D. M. (1997). Role of personality self-organization in development of mental order and disorder. Development and Psychopathology, 9, 881-906. doi: 10.1017/S095457949700148X

Cloninger, C. R., Zohar, A. H., Hirschmann, S. y Dahan, D. (2012). The psychological costs and benefits of being highly persistent: Personality profiles distinguish mood disorders from anxiety disorders. Journal of Affective Disorders, 136, 758-766. doi: 10.1016/j.jad.2011.09.046

Cohen, S., Kamarck, T. y Mermelstein, R. (1983). A global measure of perceived stress. Journal of Health \& Social Behavior, 24, 385396.

Dickman, S. J. (1990). Functional and dysfunctional impulsivity: Personality and cognitive correlates. Journal of Personality and Social Psychology, 58, 95-102. doi: 10.1037/0022-3514.58.1.95

Ernst, M. y Fudge, J. (2009). A developmental neurobiological model of motivated behavior: anatomy, connectivity and ontogeny of the triadic nodes. Neuroscience \& Biobehavioral Reviews, 33, 367382. doi: 10.1016/j.neubiorev.2008.10.009

Goldman, M. S. (1990). Experience-dependent neuropsychological recovery and the treatment of chronic alcoholism. Neuropsychological Review, 1, 75-101. doi: 10.1007/BF01108859

Goekoop, J. G., De Winter, R. F. P. y Goekoop, R. (2011). An increase of the character function of self-directedness is centrally involved in symptom reduction during remission from major depression. Depression Research and Treatment, 2011, ID 749640, 8 pgs. doi:10.1155/2011/749640

Grucza, R. A. y Goldberg, L. R. (2007). The comparative validity of 11 modern personality inventories: predictions of behavioral acts, informant reports, and clinical indicators. Journal of Personality Assessment, 89, 167-187. doi:10.1080/00223890701468568

Jones, A., Donmall, M., Millar, T., Moody, A., Weston, S., Anderson, T., Gittins, M., Abeywardana, V. y D'Souza, J. (2009). The Drug Treatment Outcomes Research Study (DTORS): Final outcomes report. Londres: Home Office. Recuperado de: http://www.dtors. org.uk/reports/DTORS_Final_Main.pdf.

Lemenager, T., Richter, A., Reinhard, I., Gelbke, J., Beckmann, B., Heinrich, M., Kniest, A., Mann, K. y Hermann, D. (2011). Impaired decision making in opiate addiction correlates with anxiety and self-directedness but not substance use parameters.
Journal of Addiction Medicine, 5, 203-213. doi: 10.1097/ ADM.0b013e31820b3e3d

Liston, C., McEwen, B. S. y Casey, B. J. (2009). Psychosocial stress reversibly disrupts prefrontal processing and attentional control. PNAS, 106, 912-917. doi: 10.1073/pnas.0807041106

Llanero-Luque, M., Ruiz-Sánchez de León, J. M., Pedrero-Pérez, E. J., Olivar-Arroyo, A., Bouso-Saiz, J. C., Rojo-Mota, G. y Puerta García, C. (2008). Sintomatología disejecutiva en adictos a sustancias en tratamiento mediante la versión española del cuestionario disejecutivo (DEX-Sp). Revista de Neurología, 47, 457-463.

Lopez-Quintero, C., Hasin, D. S., Pérez de Los Cobos, J., Pines, A., Wang, S., Grant, B. F. y Blanco, C. (2011). Probability and predictors of remission from life-time nicotine, alcohol, cannabis or cocaine dependence: results from the National Epidemiologic Survey on Alcohol and Related Conditions. Addiction, 106, 657-669. doi: 10.1111/j.1360-0443.2010.03194.x

Montag, C., Jurkiewicz, M. y Reuter, M. (2010). Low self-directedness is a better predictor for problematic internet use than high neuroticism. Computers in Human Behavior, 26, 1531-1535. doi: 10.1016/j.chb.2010.05.021

Noël, X., Brevers, D., Bechara, A., Hanak, C., Kornreich, C., Verbanck, P. y Le Bon, 0. (2011). Neurocognitive determinants of novelty and sensation-seeking in individuals with alcoholism. Alcohol and Alcoholism, 46, 407-415. doi: 10.1093/alcalc/agr048

OEDT (2011). Informe Anual 2011: El problema de la drogodependencia en Europa. Luxemburgo: Oficina de Publicaciones de la Unión Europea. doi: 10.1093/alcalc/agr048

Olivar-Arroyo, Á. (2011). Aplicaciones de la neurociencia de las adicciones en los modelos preventivos. Trastornos Adictivos, 13(Supl. 1), S20-S26.

Paris, J. (2005). Neurobiological dimensional models of personality: A review of the models of Cloninger, Depue, and Siever. Journal of Personality Disorders, 19, 156-170. doi: 10.1521/ pedi.19.2.156.62629

Pedrero-Pérez, E. J. (2009a). TCl-R-67: versión abreviada del TCI-R de Cloninger. Proceso de creación y administración a una muestra de adictos a sustancias en tratamiento. Trastornos Adictivos, 11, 12-23. doi: 10.1016/S1575-0973(09)71375-3

Pedrero-Pérez, E. J. (2009b). Evaluación de la impulsividad funcional y disfuncional en adictos a sustancias mediante el Inventario de Dickman. Psicothema, 21, 585-591.

Pedrero-Pérez, E. J., López-Durán, A. y Olivar-Arroyo, A. (2011). Addiction: frontal personality change but not personality disorder comorbidity. Implications for treatment of addictive disorders. En: Jordan, ME (Ed.), Personality Traits Theory, Testing and Influences (pp. 1-36). New York: Nova Science Publishers.

Pedrero-Pérez, E. J. y Olivar-Arroyo, Á. (2010). Estrés percibido en adictos a sustancias en tratamiento mediante la escala de Cohen: propiedades psicométricas y resultados de su aplicación. Anales de Psicología, 26, 302-309.

Pedrero-Pérez, E. J. y Rojo-Mota, G. (2008). Diferencias de personalidad entre adictos a sustancias y población general. 
Estudio con el TCl-R de casos clínicos con controles emparejados. Adicciones, 20, 251-262.

Pedrero-Pérez, E. J., Rojo-Mota, G., Ruiz-Sánchez de León, J. M., Llanero-Luque, M. y Puerta-Garcia, C. (2011). Rehabilitación cognitiva en el tratamiento de las adicciones. Revista de Neurología, 52, 163-172.

Pedrero-Pérez, E. J., Ruiz-Sánchez de León, J. M., Lozoya-Delgado, P., Llanero-Luque M., Rojo-Mota, G. y Puerta-García, C. (2011c). Evaluación de los sintomas prefrontales: propiedades psicométricas y datos normativos del cuestionario disejecutivo (DEX) en una muestra de población española. Revista de Neurología, 52, 394-404.

Pedrero-Pérez, E. J., Ruiz-Sánchez de León, J. M., Olivar-Arroyo, Á., Bouso-Saiz, J. C., Rojo-Mota, G., Llanero-Luque, M. y PuertaGarcia, C. (2009). Versión española del Cuestionario Disejecutivo (DEX-Sp): propiedades psicométricas en adictos y población no clínica. Adicciones, 21, 155-166.

Pedrero-Pérez, E. J., Ruiz-Sánchez de León, J. M., Olivar-Arroyo, Á., Rojo-Mota, G., Llanero-Luque, M. y Puerta-Garcia, C. (2011b). Diferencias de personalidad entre adictos al alcohol y controles emparejados: relación con sintomatología frontal y subtipos de adictos. Psicothema, 23, 100-106.

Pedrero-Pérez, E. J., Ruiz-Sánchez de León, J. M., Rojo-Mota, G., Llanero-Luque, M. y Puerta-García, C. (2012). Caracterización neuropsicológica de la impulsividad funcional y disfuncional en adictos a sustancias: implicaciones clínicas. Adicciones, 24, 51-58.

Pérez de los Cobos, J., Siñol, N., Bañulus, E., Batlle, F., Tejero, A. y Trujols, J. (2010). Personality traits of cocaine-dependent patients associated with cocaine-positive baseline urine at hospitalization. American Journal of Drug and Alcohol Abuse, 36, 52-56. doi: http://dx.doi.org/10.3109/00952990903572225

Reich, J. (2002). Clinical correlates of stress-induced personality disorder. Psychiatric Annals, 32, 581-588.

Richter, J. y Eisemann, M. (2002). Self-directedness as a cognitive feature in depressive patients. Personality and Individual Differences, 32, 1327-1337. doi: 10.1016/S0191-8869(01)00121-0

Rojo-Mota, G., Iraurgi-Castillo, I. y Sánchez-Cabeza. (2011). Rehabilitación cognitiva en el tratamiento de las adicciones (pp. 277-298). En Pedrero-Pérez, E. J. (coord.), Neurociencia y Adicción. Madrid: Sociedad Española de Toxicomanías.

Rosenbaum, P. R. (1989). Optimal matching for observational studies. Journal of the American Statistical Association, 84, 1024-1032. doi:10.1080/01621459.1989.10478868

Ruiz-Sanchez de León, J. M., Pedrero-Pérez, E. J., Olivar-Arroyo, Á, Llanero-Luque, M., Rojo-Mota, G. y Puerta-Garcia, C. (2010). Personalidad y sintomatología frontal en adictos y población no clínica: hacia una neuropsicología de la personalidad. Adicciones, 22, 233-244.

Schwabe, L., Dickinson, A. y Wolf, O. T. (2011). Stress, habits, and drug addiction: a psychoneuroendocrinological perspective. Experimental and Clinical Psychopharmacology, 19, 53-63. doi: $10.1037 / \mathrm{a} 0022212$
Svrakić, D., Cloninger, C. R., Svrakić, N., Lazić B., Milivojević D. y Nastasić, P. (2010). Drug addiction and choice of drugs: Temperament and personality as risk factors. Serbian Journal of Experimental and Clinical Research, 11, 93-98.

Svrakic, D. M., Draganic, S., Hill1, K., Bayon, C., Przybeck, T. R. y Cloninger, C. R. (2002). Temperament, character, and personality disorders: etiologic, diagnostic, treatment issues. Acta Psychiatrica Scandinavica, 106, 189-195. doi: 10.1034/j.16000447.2002.02196.x

Wilson, B. A., Alderman, N., Burgess, P. W., Emslie, H. y Evans, J. J. (1996). Behavioural assessment of the Dysexecutive Syndrome. Bury St. Edmunds, UK: Thames Valley Test Company. 